\title{
Regulação Metabólica e Produção de Espécies Reativas de Oxigênio Durante a Contração Muscular: Efeito do Glicogênio na Manutenção do Estado Redox Intracelular
}

\author{
Metabolic Regulation and Production of Oxygen Reactive Species \\ During Muscule Contraction: Effect of Glycogen on Intracellular \\ Redox State
}

\author{
Leonardo R. Silveira \\ Sandro M. Hirabara' \\ Rafael H. Lambertucci ${ }^{1}$ \\ Carol V. leandro, Jarlei Fiamoncini² \\ Carlos HJ Pinheiro ${ }^{2}$ \\ Anielle C. A. D'Angelo² \\ Reinaldo A. Bassit ${ }^{2}$ \\ Tânia C Pithon-Curi² \\ Rui Curi²
}

\section{Departamento de Fisiologia e Biofísica, Instituto de Ciências Biomédicas, Universidade de São Paulo. \\ 2. Universidade Federal de Pernambuco, Centro de Ciências da Saúde, Departamento de Nutrição. \\ Endereço para correspondência: Departamento de Fisiologia e Biofísica, Instituto de Ciências Biomédicas, Universidade de São Paulo, Cidade Universitária, Av. Professor Lineu Prestes, 1524 Butantã, São Paulo, SP 05508-900, Brasil. Tel.: 113091 7245; \\ fax: 1130917285. \\ E-mail: Irs@icb.usp.br.}

Submetido em 31/01/2007 Versão final recebida em 15/06/2007 Aceito em 26/07/2007

\begin{abstract}
RESUMO
O exercício físico prolongado reduz os estoques de glicogênio muscular. Nessas condições, os processos de fadiga muscular são estimulados coincidindo com um aumento na produção de espécies reativas de oxigênio. A suplementação de carboidratos ou de antioxidantes isoladamente contribui para a melhora da performance muscular, sugerindo um efeito importante da depleção de substrato (glicose) e do aumento da produção de EROs no desenvolvimento da fadiga muscular durante a atividade física. Embora o mecanismo seja desconhecido, estamos propondo neste estudo que uma maior disponibilidade de glicogênio poderia favorecer uma maior atividade da via das pentoses fosfato, aumentando a disponibilidade de NADPH e GSH no tecido muscular esquelético. Uma maior capacidade antioxidante aumentaria a capacidade do tecido muscular em atividade, mantendo o equilíbrio redox durante atividade física prolongada e melhorando o desempenho. Neste processo, o ciclo glicose-ácido graxo pode ser importante aumentando a oxidação de lipídio e reduzindo o consumo de glicogênio durante a atividade prolongada. Além disso, um aumento na produção de EROs pode reduzir a atividade de enzimas importantes do metabolismo celular incluindo a aconitase e a a-cetoglutarato desidrogenase, comprometendo a produção de energia oxidativa, via predominante na produção de ATP durante a atividade muscular prolongada.
\end{abstract}

Palavras-chave: fadiga, antioxidantes, metabolismo, músculo esquelético, exercício prolongado.

\section{ABSTRACT}

Fatigue is closely related to the depletion of glycogen in the skeletal muscle during prolonged exercise. Under this condition, the production of oxygen reactive species (ROS) is substantially increased. It has been shown that dietary supplementation of carbohydrate or antioxidant attenuates muscle fatigue during contraction. This suggests that glycogen availability and/or elevated ROS production plays an important role on muscle fatigue development during prolonged muscle activity. Although the mechanism is still unknown, we propose that elevated muscle glycogen availability may lead to a high activity of hexose monophosphate pathway, increasing the NADPH and glutathione concentration in the skeletal muscle tissue. Elevated antioxidant capacity would increase the muscle redox balance during muscle contraction, improving performance. In this process, the glucose-fatty acid cycle may be important to increase lipid oxidation and consequently decrease glycogen utilization during prolonged activity. In addition, an elevated ROS production could reduce the activity of key metabolic enzymes including aconitase and a-ketoglutarate dehydrogenase, decreasing the oxidative energy production in the skeletal muscle during prolonged activity.

Keywords: fatigue, antioxidants, metabolism, skeletal muscle, prolonged exercise

\section{INTRODUÇÃO}

O papel protetor da glutationa como antioxidante durante a atividade física tem sido extensivamente investigado. Há muitas evidências de que a glutationa é um dos principais antioxidantes do tecido muscular esquelético exercendo função vital na manutenção do estado redox intracelular durante a contração $0^{(1-3)}$ e protegendo este tecido contra o ataque oxidativo imposto pela elevada taxa metabólica durante o exercício ${ }^{(3,4)}$. Em adição, várias proteínas incluindo proteínas quinases, fatores de transcrição e enzimas do metabolismo celular possuem propriedades redox sensitivas em resíduos de cisteína ricos em grupos tióis(2). Essas propriedades parecem exercer função sinalizadora importante mediada por mecanismos sensíveis à modulação redox, ${ }^{(2,5,6)}$. Assim, o estado redox dos grupos SH de um amplo espectro de proteínas intracelulares é regulado por um fino mecanismo mediado por glutationa, tiorredoxina e possivelmente outros antioxidantes associados (Figura 1).

Esta função é especialmente importante durante a atividade muscular extenuante onde a produção de espécies reativas de oxigênio é substancialmente aumentada na medida em que o processo de fadiga aproxima-se $\mathrm{e}^{(7-9)}$. Durante o exercício prolongado, a ocorrência de fadiga 


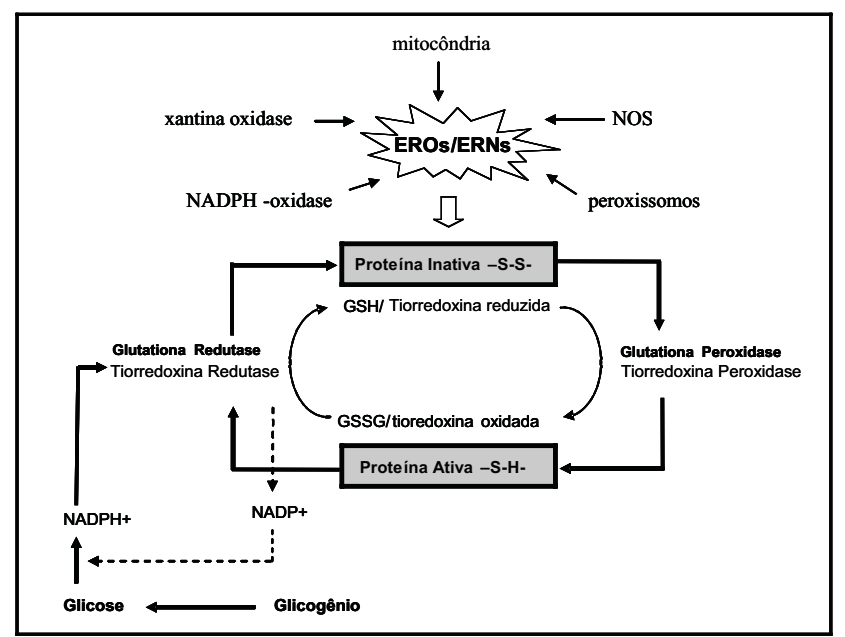

Figura 1. Efeito do sistema glutationa/tiorredoxina peroxidase na manutenção do estado redox (-SH-) de proteínas intracelulares suscetíveis a oxidação por Espécies reativas de oxigênio (espécies reativas de oxigênio) e ERNs (espécies reativas de nitrogênio). NOS, óxido nítrico sintase; GSH, glutationa reduzida; GSSG, glutationa oxidada; $\mathrm{NADPH}^{+}$, nicotinamida adenina dinucleotídio fosfato reduzida; NADP+, nicotinamida adenina dinucleotídio fosfato oxidada.

coincide com a depleção dos estoques de glicogênio muscular ${ }^{(10,11)}$. Este fato sugere uma associação entre a redução dos estoques intramusculares de glicogênio e o aumento de espécies reativas de oxigênio na fadiga muscular. Embora o mecanismo pelo o qual a produção de espécies reativas de oxigênio é acelerada durante contrações intensas ainda não seja totalmente conhecido, uma possível relação causaefeito suporta essa associação. Por exemplo, em preparações in vitro usando músculos isolados, foi mostrado que a adição de antioxidantes exógenos aumenta a força de contração durante a atividade muscular intensa ${ }^{(8,12)}$. Em adição, o efeito positivo de antioxidantes in vivo indica que uma produção elevada de espécies reativas de oxigênio limita a capacidade de trabalho muscular durante o exercício prolongado (endurance), constituíndo-se em um componente importante no processo de fadiga ${ }^{(9)}$. Nashiwati et al. ${ }^{(12)}$ demonstraram que a infusão de oxidantes no tecido muscular durante a atividade física acelera os processos de fadiga. Embora o mecanismo pelo qual esse aumento na produção de espécies reativas de oxigênio durante o exercício exaustivo seja desconhecido, a redução na capacidade antioxidante intracelular pode estar envolvida.

No tecido muscular esquelético, a oxidação de glutationa excede a capacidade intracelular de reoxidação, resultando em aumento na razão glutationa oxidada/glutationa reduzida ${ }^{(1,3,4)}$. Nessas condições, o fígado aumenta a liberação de glutationa para o sangue, elevando a captação e a capacidade antioxidante do tecido muscular durante o processo de contração. A glutationa é predominantemente regenerada no fígado e músculo esquelético às custas de $\mathrm{NADPH}^{+}$(nicotinamida adenina dinucleotídio), um dos produtos da via das pentoses ${ }^{(13,14)}$. $\mathrm{NADPH}^{+}$é utilizado como substrato pela enzima glutationa redutase, gerando como produto glutationa, o principal substrato para o sistema glutationa peroxidase (Figura 2). Porém, é importante destacar que esta via é regulada pelas concentrações de glicose 6-P, um intermediário da via glicolítica, cuja concentração é mantida às custas da degradação de glicogênio(15). Com base nesse mecanismo, nossa hipótese é de que uma redução nos estoques endógenos de glicogênio durante o exerćcio prolongado levaria a uma consequente redução nas concentrações de glicose-6-fosfato, diminuindo a disponibilidade de substrato para a via das pentoses, comprometendo a regeneração de glutationa. Dessa forma, estamos propondo neste estudo, um mecanismo metabólico adicional, pelo qual o aumento da produção de espécies reativas de oxigênio aceleraria os processos de fadiga durante contrações musculares intensas.

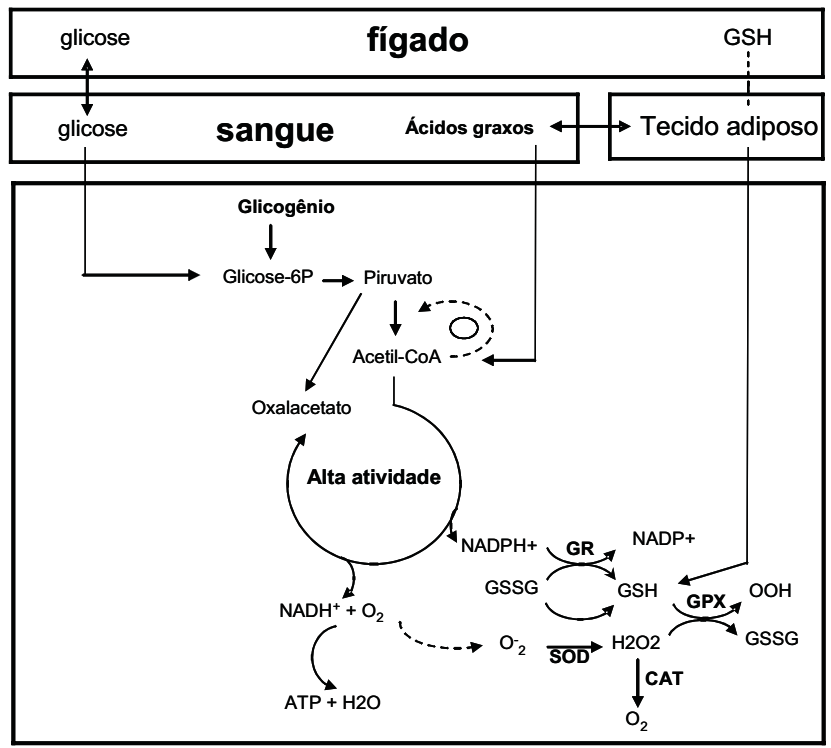

Figura 2. Efeito da glicose plasmática e de glutationa hepático na manutenção do estado redox intracelular. As abreviaturas são: $\mathrm{NADH}^{+}$, nicotinamida adenina dinucleotídio reduzida; $\mathrm{NAD}^{+}$, nicotinamida adenina dinucleotídio oxidada; $\mathrm{GSH}$, glutationa reduzida; GSSG, glutationa oxidada; $\mathrm{NADPH}^{+}$, nicotinamida adenina dinucleotídio fosfato reduzida; $\mathrm{NADP}^{+}$, nicotinamida adenina dinucleotídio fosfato oxidada; CAT, catalase; $\mathrm{GPX}$, glutationa peroxidase; $\mathrm{GR}$, glutationa redutase; $\mathrm{SOD}$, superóxido dismutase; $\mathrm{O}_{2}^{-}$ ânion superóxido; $\mathrm{H}_{2} \mathrm{O}_{2}$, peróxido de hidrogênio; $\mathrm{OOH}$, hidroperóxidos.

\section{O Glicogênio Como Regulador da Produção de Espé- cies Reativas de Oxigênio}

Há mais de 60 anos, há evidências de que o aumento das concentrações endógenas de glicogênio antes do exercício eleva a capacidade do atleta de realizar atividade física de longa duração ${ }^{(16,17)}$. Durante esse tipo de exercício, a ocorrência de fadiga é fortemente correlacionada com a depleção do glicogênio muscular ${ }^{(18,19)}$. Nessas condições, o músculo esquelético exibe concentração elevada de inosina 5'-monofosfato (IMP) e amônia $\left(\mathrm{NH}^{+}\right)$, sugerindo um aumento da razão ADP/ATP(10,11). Apesar do consenso de que a depleção do glicogênio favorece a fadiga muscular, ainda existem poucas evidências do mecanismo pelo qual esse processo ocorre. A redução nas concentrações endógenas de glicogênio diminui a atividade da via glicolítica, responsável pela manutenção do fluxo de substratos para o ciclo de Krebs (acetil-CoA, oxalacetato e $\alpha$-cetoglutarato), seguido de redução de $\mathrm{NADH}$ e $\mathrm{FADH}_{2}$, importantes fornecedores de elétrons para a síntese oxidativa de ATP mitocondrial(11,20,21).

Em adição a esse mecanismo, um menor conteúdo de glicogênio muscular e hepático reduziria a disponibilidade de G6-P, um importante intermediário da via glicolítica e responsável pela manutenção do fluxo de substratos para a via das pentoses ${ }^{(15)}$. Essa via é a principal fornecedora de elétrons para a regeneração de $\mathrm{NADPH}^{+}$, o principal doador de elétrons e substrato da enzima glutationa redutase na redução de glutationa oxidada em glutationa ${ }^{(23)}$. Nessas condições, a disponibilidade de glutationa fica reduzida favorecendo a alteração do estado redox intracelular para um estado mais oxidado, o que poderia levar ao aumento na oxidação dos grupos tióis de proteínas com funções sinalizadoras importantes durante a atividade muscular ${ }^{(1-3)}$, incluindo enzimas do metabolismo de glicose, ácidos graxos e aminoácidos (figura 3).

Em suporte a essa proposta, Vina et al.(24), comparando valores pré com os de pós- exercício exaustivo, verificaram em ratos e humanos um aumento nas concentrações sanguíneas de glutationa oxidada de até 3,5 vezes. Embora informações do estado antioxidante da razão glutationa/ glutationa oxidada no tecido muscular humano durante o exercício seja limitado, foi demonstrado em ratos que a atividade muscular aumenta a oxidação de glutationa. Lew et al. ${ }^{(25)}$ mostraram que o exercício exaustivo consistentemente reduz as concentrações de glutationa em músculo e 


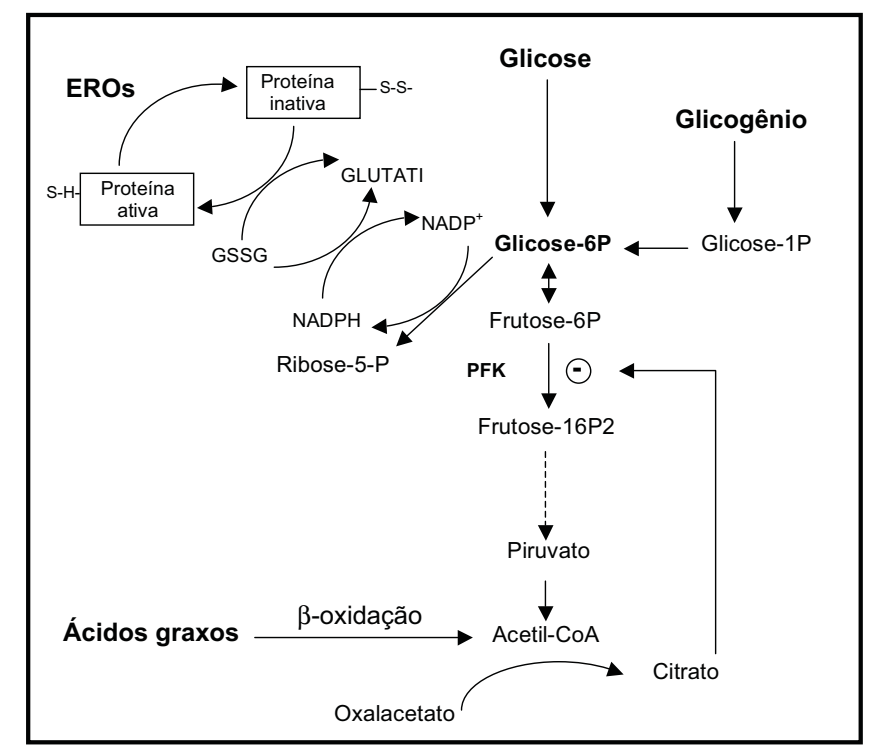

Figura 3. Efeito da inibição da via glicolítica, da disponibilidade de glicose e do glicogênio intracelular como precursores de $\mathrm{NADPH}^{+}$e GSH na manutenção do estado redox de proteínas intracelulares. As abreviações são: GSH, glutationa reduzida; GSSG, glutationa oxidada; $\mathrm{NADPH}^{+}$, nicotinamida adenina dinucleotídio fosfato reduzida; NADP+, nicotinamida adenina dinucleotídio fosfato oxidada; PFK. Fosfofrutoquinase; S-S-, ponte disulfeto; S-H-, grupo sulfidrila.

fígado durante corrida em esteira. Portanto, podemos assumir com base nos achados descritos acima, que o exercício físico aumenta a oxidação de glutationa no sangue, músculo e fígado.

Quando a oxidação de glutationa é baixa, grande parte da glutationa oxidada produzida no tecido muscular pode ser rapidamente regenerada pelo sistema glutationa redutase/NADPH+(3,4,24). Porém, alterações do balanço redox para um estado mais oxidado pode comprometer a capacidade desse sistema, favorecendo a ocorrência de estresse oxidativo ${ }^{(2,3,7,26)}$. Nossa proposta é de que essa condição seja favorecida durante o exercício exaustivo prolongado onde comprovadamente ocorre redução dos estoques endógenos de glicogênio. Nessa condição, a disponibilidade de G6-P fica diminuída reduzindo a capacidade de produção de $\mathrm{NADPH}^{+}$pela via das pentoses, comprometendo a atividade do sistema glutationa redutase/ $\mathrm{NADPH}^{+}$na regeneração de glutationa, um dos principais antioxidantes intracelulares e substrato da enzima glutationa peroxidase ${ }^{(1,2)}$. Embora na maioria dos tecidos a catalase é considerada o principal seqüestrador do $\mathrm{H}_{2} \mathrm{O}_{2}$, no tecido muscular esquelético, a glutationa peroxidase parece exercer essa função com maior eficiência ${ }^{(27)}$. No tecido muscular, estas enzimas apresentam diferentes valores de afinidade pelo substrato $\mathrm{H}_{2} \mathrm{O}_{2}(\mathrm{Km})$, sendo o valor de $\mathrm{km}$ da catalase mais elevado ${ }^{(28)}$. Portanto, no músculo esquelético, a glutationa peroxidase parece ser a primeira linha de defesa por apresentar maior afinidade pelo $\mathrm{H}_{2} \mathrm{O}_{2}$, demonstrando a importância do glicogênio muscular como substrato da via das pentoses na regeneração de glutationa.

\section{Efeito do Ciclo Glicose-Ácido Graxo na Regeneração de Glu- tationa}

Os ácidos graxos, por apresentarem uma estrutura altamente rica em elétrons (reduzida), possuem alta capacidade de produzir energia oxidativa $^{(16,17)}$. Nesse processo, os ácidos graxos induzem uma fina regulação no metabolismo de carboidratos, reduzindo a oxidação de glicose ${ }^{(29)}$. Em condições de oferta lipídica elevada, como verificado no jejum ou demonstrado originalmente em músculo esquelético, cardíaco e no tecido hepático, este mecanismo é mantido por uma elevada razão das concentrações de acetil-CoA/CoA-SH, como conseqüência de uma alta taxa de oxidação de ácidos graxos, seguido de aumento no conteúdo intracelular de citrato e glicose-6-fosfato. O acúmulo de
acetil-CoA reduz a atividade do complexo piruvato desidrogenase (PDH), via ativação da piruvato desidrogenase quinase, a enzima responsável pela fosforilação e inativação do complexo, reduzindo a oferta de piruvato (glicose) como substrato oxidativo. De modo sinérgico, o conteúdo elevado de citrato inibe a atividade da enzima reguladora da via glicolítica, a fosfofrutoquinase (PFK) $(16,17,29)$ (figura 4). Este efeito aumenta a razão glicose-6.fosfato/frutose 1,6-fosfato-2, o que favorece o desvio do fluxo de metabólitos em direção à síntese de $\mathrm{NADPH}^{+}$pela via das pentoses (Figura 3).

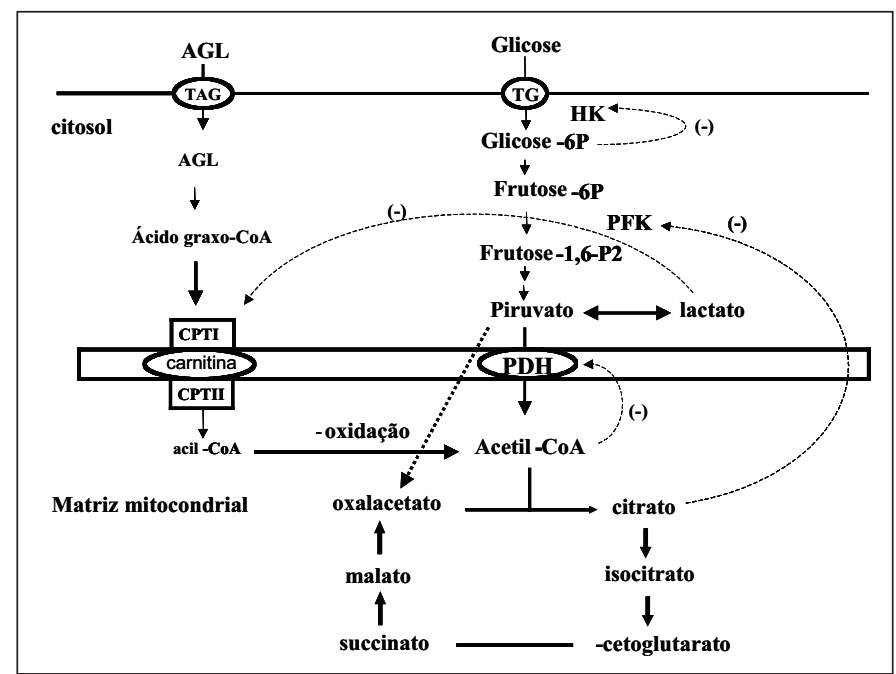

Figura 4. Efeito do ciclo glicose-ácido graxo na redução do metabolismo de glicose e aumento na disponibilidade de glicose-6P para manutenção das concentrações de NADPH e GSH intracelular. AGL, ácido graxo livre; TAG, transportador de ácidos graxos; TG, transportador de glicose; HK, hexoquinase; PFK, fosfofrutoquinase; CPT, carnitina palmitoiltransferse; $\mathrm{PDH}$, piruvato desidrogenase.

Este conceito (ciclo glicose-ácido graxo) explica a maior utilização dos ácidos graxos pelo tecido muscular durante a atividade física moderada mantida por período prolongado ${ }^{(16,17,30)}$. Porém, considerando o limitado estoque endógeno de carboidratos em nosso organismo, o aumento na disponibilidade e oxidação de ácidos graxos é importante não somente para a produção de ATP, mas também para a redução na utilização de glicogênio(16). Caso essa regulação não ocorra, os estoques de glicogênio podem ser depletados precocemente, comprometendo a performance muscular ${ }^{(16,17)}$. Igualmente, observações de que durante situações de estresse, incluindo o exercício físico, o aumento na produção de espécies reativas de oxigênio sempre excede a capacidade de defesa antioxidante ${ }^{(1,26,31)}$, aumenta a possibilidade de o conteúdo intracelular de glutationa e favorece a melhora da performance durante o exercício prolongado. Em adição, estamos propondo que essa importante estratégia metabólica de economia do glicogênio hepático e muscular para manutenção da performance durante atividades prolongadas está associada a maior disponibilidade de glutationa. Nessas condições, uma disponibilidade elevada de acetil-CoA proveniente da oxidação de ácidos graxos leva a aumento de citrato, um conhecido inibidor da fosfofrutoquinase, principal enzima reguladora da via glicolítica. Aumentando, dessa forma, a oferta de glicose-6-fosfato para a via das pentoses. Com base na regulação metabólica proposta acima, poderíamos assumir, portanto, que o efeito positivo do ciclo glicose-ácido graxo na performance está associado à manutenção do estado redox intracelular do músculo esquelético via aumento na razão glutationa/ glutationa oxidada durante o exercício prolongado.

\section{Anaplerose e Produção de Espécies Reativas de Oxigênio}

Embora a via das pentoses seja um importante fornecedor de NADPH em diferentes tecidos, sua atividade é mais alta em tecidos com capacidade elevada de síntese de lipídio, como é o caso do fígado e dos adipócitos ${ }^{(15)}$. O músculo esquelético, embora apresente uma 
atividade relativamente menor, pode compensar essa diferença por aumento alternativo na atividade da enzima isocitrato desidrogenase, responsável pela conversão de isocitrato + $\mathrm{NADP}^{+}$em $\alpha$-cetoglutarato $+\mathrm{NADPH}^{+(23,32)}$. A concentração de isocitrato, bem como a de outros intermediários do ciclo de Krebs, é mantida às custas de acetil-CoA e oxaloacetato, produtos da oxidação de ácidos graxos e glicose ${ }^{(15)}$. A principal função do ciclo de Krebs no músculo esquelético é de oxidar acetil-CoA produzindo $\mathrm{NADH}^{+}$e $\mathrm{FADH}_{2}$ para a geração oxidativa de ATP mitocondrial, além de gerar intermediários para a síntese de outras moléculas ${ }^{(10,11,15)}$.

Embora o mecanismo completo pelo qual a redução das concentrações de glicogênio reduz a performance seja apenas parcialmente conhecido(19,20,33), é possível que uma redução nas concentrações de intermediários do ciclo de Krebs esteja envolvido. Um aumento líquido nas concentrações dos intermediários do ciclo de Krebs é necessário para o estabelecimento e manutenção de uma taxa elevada de fluxo de substratos através deste, aumentando a síntese de ATP(10,11,15,19,20). O aumento das concentrações dos intermediários do ciclo de Krebs, portanto, é dependente de várias reações anapleróticas (aumento das concentrações de intermediários do ciclo de Krebs) incluindo o piruvato como principal substrato ${ }^{(15)}$. Em contraste, durante o exercício prolongado, o conteúdo de glicogênio muscular é reduzido, seguido de um declínio gradual do conteúdo dos intermediários do ciclo de Krebs, o que levaria a um comprometimento do fluxo de substratos pelo ciclo e da geração de ATP mitocondrial ${ }^{(10,11,20)}$. Há evidências de que a menor disponibilidade de substrato para o ciclo de Krebs reduz a síntese de ATP mitocondrial e conseqüentemente a performance ${ }^{(10,20)}$. Uma redução nas concentrações dos intermediários do ciclo de Krebs pode estimular as reações anapleróticas numa tentativa de aumentar a disponibilidade desses intermediários e conseqüentemente a síntese de energia oxidativa ${ }^{(11,15,20,34,35)}$. Nesse contexto, diferentes mecanismos anapleróticos controlados por reações de carboxilação e descarboxilação podem controlar o fluxo que regula a entrada e saída de carbonos do ciclo. Entre estes mecanismos, inclui-se as reações do ciclo das purinas ${ }^{(36)}$ e a reação catalisada pela glutamato desidrogenase ${ }^{(37)}$ (Figura 5). Esses mecanismos permitem ajustar as concentrações dos intermediários do ciclo de Krebs em resposta a demanda metabólica.

Um aumento da atividade anaplerótica pelas vias acima descritas poderia contribuir com o aumento do fluxo de entrada de substratos no ciclo de $\operatorname{Krebs}^{(11,20,35,36,38)}$, favorecendo a atividade da isocitrato desidrogenase e consequentemente a síntese de $\mathrm{NADPH}^{+}$, importante doador de elétrons para a regeneração de glutationa no músculo ${ }^{(32)}$. Em adição, o fluxo de substratos no ciclo de Krebs pode ser regulado pelo aumento de ADP/ATP, $\mathrm{Ca}^{++}$e NAD/NADH ${ }^{+}$, importantes moduladores

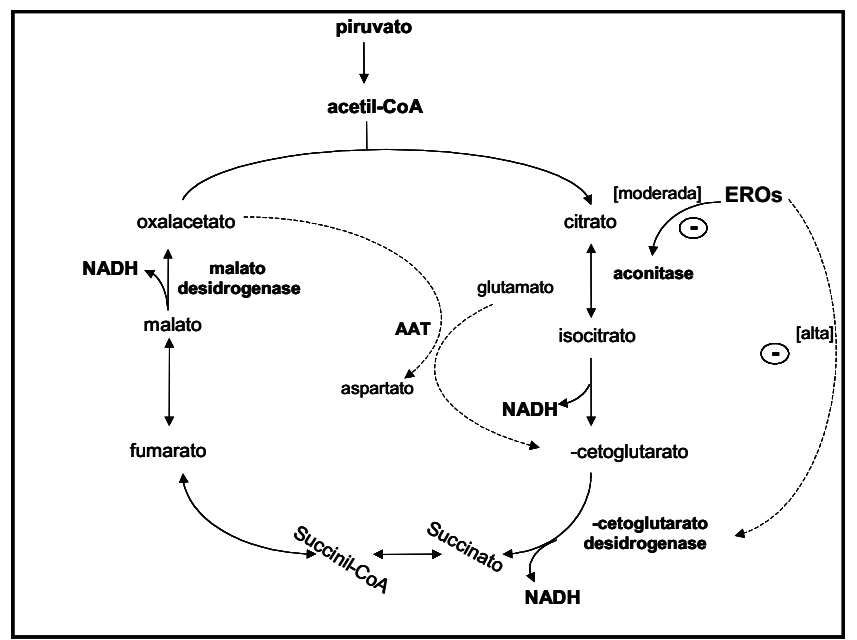

Figura 5. Efeito inibitório das Espécies reativas de oxigênio na atividade da aconitase e $\alpha$-cetoglutarato desidrogenase durante a atividade muscular. AAT, aspartato aminotransferase; $\mathrm{NADH}^{+}$, nicotinamida adenina dinucleotídio reduzida; EROs, espécies reativas de oxigênio. do complexo piruvato desidrogenase ${ }^{(15)}$, e das enzimas reguladoras do ciclo (non-equilibrium, $\Delta \mathrm{G}$-), citrato sintase, isocitrato desidrogenase e $\alpha$-cetoglutarato desidrogenase. Igualmente ao efeito da produção elevada de oxidantes na diminuição da força de contração do músculo esquelético in vitro citado anteriormente ${ }^{(12)}$, Taegtmeyer et al. ${ }^{(39)}$ demonstraram em músculo cardíaco, que uma redução da concentração dos intermediários do ciclo de Krebs reduz a força de contração, um efeito que é revertido pela adição de substratos anapleróticos. Assim, um aumento na disponibilidade dos intermediários do ciclo de Krebs, bem como na atividade das enzimas reguladoras do ciclo, podem aumentar a síntese de $\mathrm{NADPH}^{+}$, contribuindo com a regeneração de glutationa. Portanto, considerando a importância da glutationa como antioxidante endógeno no tecido muscular durante a atividade, podemos especular que estratégias que visam o aumento do fluxo de entrada de intermediários do ciclo de Krebs, incluindo a suplementação de carboidratos ou a reposição de substratos anapleróticos como aminoácidos de cadeia ramificada, glutamina e outros aminoácidos (Figura 6), podem favorecer a síntese de energia oxidativa, bem como regular a produção de espécies reativas de oxigênio durante o processo de contração muscular via aumento nas concentrações de glutationa.

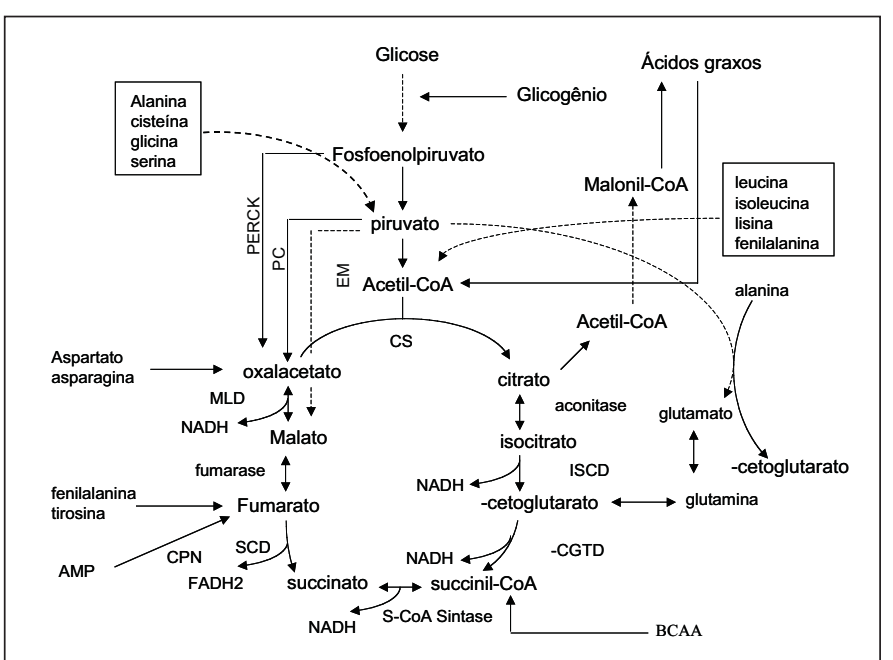

Figura 6. Vias de aumento de intermediários do ciclo de Krebs (anaplerose). PEPCK, fosfoenol piruvato quinase; PC, piruvato carboxilase; EM, enzima málica; CS, citrato sintase; ISCD, isocitrato desidrogenase; $\alpha$-CGTD, $\alpha$-cetoglutarato desidrogenase; $S$ CoA sintase; succinil-CoA sintase; SCD, succinato desidrogenase; MLD, malato desidrogenase; AMP, adenosina monofosfato; CPN, ciclo das purinas nucleotídios; BCAA, aminoácidos de cadeia ramificada.

\section{As Espécies Reativas Como Reguladores Metabólicos}

Embora o mecanismo pelo o qual a produção de espécies reativas de oxigênio são aumentada durante a fadiga seja desconhecido, a regulação metabólica mitocondrial imposta pelo elevado consumo de oxigênio exerce um efeito direto nesse processo ${ }^{(40-42)}$. A mitocôndria, um importante sítio gerador de Espécies reativas de oxigênio durante a atividade muscular ${ }^{26,31)}$, pode também ser vulnerável as ações dessas espécies ${ }^{(31,41,42)}$. Em situações nas quais a produção de Espécies reativas de oxigênio é aumentada, o superóxido tem sido descrito, ao longo dos últimos anos, como um potente inibidor da enzima aconitase, por um mecanismo envolvendo a oxidação do ferro, importante co-fator dessa enzima(41). Embora a aconitase não seja uma enzima reguladora do ciclo de Krebs $\left(\Delta \mathrm{G}^{+}\right)$, a redução na atividade dessa enzima poderia resultar em menor fluxo de substratos. Conforme descrito anteriormente, a diminuição da atividade do ciclo de Krebs poderia reduzir a disponibilidade de agentes redutores, $\mathrm{NADH}$ e $\mathrm{FADH}_{2}$, para a cadeia de transporte de elétrons mitocondrial, comprometendo a síntese de ATP. Embora ainda existam poucas informações a esse respeito, uma redução significante na atividade da aconitase foi verificada em músculo esquelético de pacientes com intorelância severa ao exercício 
físico ${ }^{(43)}$, aumentando as suspeitas de que uma redução na performance durante a atividade muscular pode ser mediada por aumento de Espécies reativas de oxigênio.

Este mecanismo, conforme proposto por Gardner e Fridovitch (44), poderia servir como retroalimentação negativa para as células musculares em situação de estresse oxidativo elevado. Isto é, uma redução da atividade mitocondrial, via redução do fluxo de substratos pelo ciclo de Krebs, reduziria a formação de Espécies reativas de oxigênio. Em contraste, a aconitase pode ser reativada pela regeneração do ferro, às custas de grupos tióis, incluindo principalmente a glutationa. Porém, em situações de consumo elevado e baixa disponibilidade de glutationa, como ocorre no exercício prolongado $(1,3,13,14)$, esse mecanismo de inibição do fluxo no ciclo de Krebs pode ser fortemente favorecido. Nessas condições, uma produção elevada de Espécies reativas de oxigênio poderia inibir a aconitase, aumentando as concentrações intracelulares de citrato ${ }^{(41)}$. Uma vez em concentração elevada, o citrato poderia reduzir o fluxo glicolítico, conforme descrito anteriormente, pelo ciclo glicose-ácido graxo ${ }^{(16,29)}$, além de exercer efeito direto na atividade da citrato sintase. Esta enzima apresenta atividade longe do equilíbrio ( $\Delta \mathrm{G}-)$ e, portanto, exerce função reguladora na atividade do ciclo de Krebs. Em outros estudos, tem sido verificado ainda que um desequilíbrio do estado redox intracelular pode favorecer a inibição de outras enzimas importantes do metabolismo durante o processo de contração muscular, como a creatina quinase ${ }^{(45)}$, gliceraldeído 3-fosfato desidrogenase ${ }^{(46)}$ e cadeia de transporte de elétrons ${ }^{(47)}$. Esse mecanismo pode explicar, pelo menos em parte, a redução da performance observada nessas condições. Porém, a disponibilidade de NADH para a cadeia de transporte de elétrons mitocondrial não é significativamente alterada mesmo quando a atividade da aconitase é totalmente inibida ${ }^{(42)}$. Interessantemente, a disponibilidade de NADH foi reduzida por inibidores da aconitase, somente quando a enzima $\alpha$-cetoglutarato desidrogenase foi paralelamente bloqueada. Essas observações nos permitem concluir que, mesmo com a inibição da aconitase, o suprimento de NADH para a cadeia de transporte de elétrons mitocondrial é mantido, provavelmente às custas de reações anapleróticas, mantidas por algum seguimento do ciclo de Krebs (Figura 6).

Igualmente importante ao efeito do superóxido, outras espécies incluindo $\mathrm{O}_{2} \mathrm{O}_{2}$, óxido nítrico e peróxinitrito podem também inibir a atividade da aconitase ${ }^{(41)}$. Um aumento nas concentrações de $\mathrm{H}_{2} \mathrm{O}_{2}$ poderia reduzir ainda a atividade de uma das principais enzimas reguladoras do ciclo de Krebs, a $\alpha$-cetoglutarato desidrogenase $\left(\Delta \mathrm{G}^{-}\right)$, seguido de um menor potencial de membrana $(\Delta \psi)$ como resultado da menor disponibilidade de NADH gerado pela baixa atividade do ciclo de Krebs ${ }^{(42)}$ (Figura 6).

Nossa hipótese, portanto, é de que um desequilíbrio nas concentrações de $\mathrm{H}_{2} \mathrm{O}_{2}$, durante a atividade muscular prolongada, imposta pela baixa disponibilidade de NADPH, substrato do sistema glutationa redutase/peroxidase poderia favorecer o acúmulo de $\mathrm{H}_{2} \mathrm{O}_{2}$ e consequentemente inibir a atividade da $\alpha$-cetoglutarato desidrogenase. Porém, em condições de produção moderada de Espécies reativas de oxigênio, a inibição parcial do ciclo de Krebs, conforme demonstrado pela inibição da aconitase, pode ser compensada por maior atividade anaplerótica, o que poderia manter o conteúdo de NADH para a cadeia de elétrons mitocondrial. Embora ainda faltem estudos conclusivos a esse respeito, existem evidências de que, em situações de reduzida disponibilidade de substrato, o fluxo para o ciclo de Krebs pode ser mantido às custas de diferentes reações anapleróticas. Essas evidências nos permite especular que nessa situação, reações anapleróticas podem gerar intermediários independentemente da atividade da aconitase, como é o caso das reações catalisadas pelas enzimas descritas abaixo:

\section{aspartato aminotransferase}

oxalacetato + glutamato $\longrightarrow \alpha$-cetoglutarato + aspartato

\section{alanina aminotransferase}

piruvato + glutamato $\longrightarrow \alpha$-cetoglutarato + alanina

\section{glutamato desidrogenase}

glutamato $+\mathrm{NAD}^{+} \longrightarrow \alpha$-cetoglutarato $+\mathrm{NADH}+\mathrm{NH}_{3}$

\section{glutamato piruvato transaminase}

glutamato + piruvato $\alpha$-cetoglutarato + alanina

\section{enzima málica}

piruvato $+\mathrm{CO}_{2}+\mathrm{NADPH} \longrightarrow \mathrm{NADP}^{+}+$malato

\section{piruvato carboxilase}

piruvato $+\mathrm{CO}_{2}+$ ATP $\longrightarrow$ ADP $+\mathrm{Pi}+$ oxalacetato

\section{fosfoenolpiruvato caboxiquinase}

fosfoenolpiruvato $+\mathrm{CO}_{2}+$ IDP $\longrightarrow$ oxalacetato + ITP

Via do ciclo das purinas de nucleotídios (Figure 7).

$$
\begin{aligned}
\mathrm{AMP} \longrightarrow \mathrm{IMP}+\mathrm{NH}_{3} \\
\mathrm{Aspartato}+\mathrm{GTP} \longrightarrow \text { Adenilsuccinato }+\mathrm{GDP}+\mathrm{Pi} \longrightarrow \\
\mathrm{AMP}+\text { fumarato }
\end{aligned}
$$

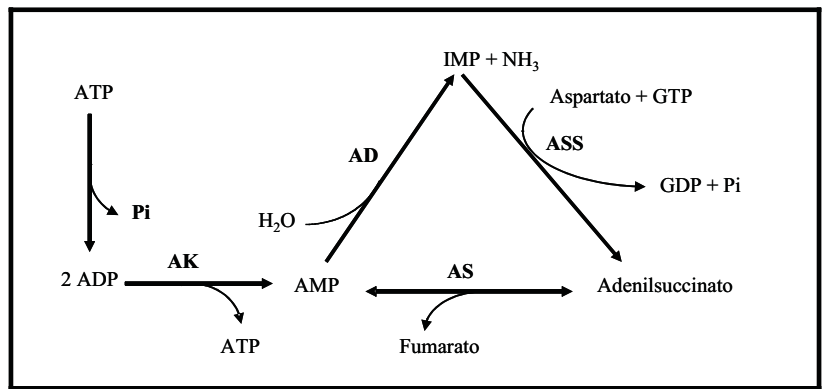

Figura 7. $O$ ciclo das purinas nucleotídios como fonte de geração de fumarato. $A D$, adenilato desaminase; ASS, adenilsuccinato sintase; AS, Adenilsuccinase; AMP, adenosina monofosfato; IMP, inosina monofosfato; AK, adenilato quinase; GTP, guanosina trifosfato; GDP, guanosina difosfato; Pi, fosfato inorgânico.

O ciclo de Krebs possui 3 etapas de formação de NADH catalisadas pelas enzimas isocitrato desidrogenase, $\alpha$-cetoglutarato desidrogenase e malato desidrogenase, além da succinato desidrogenase principal via de produção de $\mathrm{FADH}_{2}$ (Figura 5). Em adição, o complexo piruvato desidrogenase contribui nesse processo com a produção de NADH em situações de elevada demanda metabólica e disponibilidade de glicose ${ }^{(15)}$. Portanto, qualquer aumento no fluxo dessas vias de formação, independente de NADH, pode favorecer a manutenção da síntese oxidativa de ATP durante a atividade muscular prolongada. Embora as reações acima são descritas como importantes vias anapleróticas, durante o exercício prolongado ${ }^{(11,20,34-38)}$, a redução nos estoques de glicogênio pode comprometer a disponibilidade de substrato na forma de oxalacetato, piruvato e fosfoenolpiruvato para algumas dessas vias, como é o caso das reações catalisadas pela aspartato aminotransferase, alanina aminotransferase, glutamato piruvato transaminase, enzima málica e piruvato carboxilase. Dessa forma, aumentando a possibilidade da enzima glutamato desidrogenase e do ciclo das purinas de nucleotídios serem as principais vias anapleróticas durante a atividade muscular prolongada. Embora ainda haja poucas evidências desse mecanismo e da atividade dessas vias durante a atividade prolongada, a maior razão capacidade oxidativa/capacidade antioxidante nos músculos do tipo I (oxidativo) comparado a menor razão capacidade oxidativa/capacidade antioxidante nos músculos do tipo II (glicolítico) ${ }^{(48)}$ sugere que a capacidade antioxidante elevada aumentaria a eficiência do trabalho do músculo esquelético durante o exercício prolongado, favorecendo o equilíbrio do estado redox, menor comprometimento do fluxo de entrada de substratos no ciclo de Krebs e maior disponibilidade de $\operatorname{ATP}^{(1,11,41,42)}$. Ao contrário, uma menor capacidade antioxidante, como já descrito para o músculo tipo II, favoreceria maior desequilíbrio do estado redox intracelular, reduzindo a atividade mitocondrial (inibição da aconitase e $\boldsymbol{\alpha}$-cetoglutarato desidrogenase) e desviando o fluxo 
metabólico para a produção de lactato. O que seria compatível com a demanda metabólica imposta por esse tipo de músculo durante a atividade intensa.

Efeito da Suplemetação de Carboidratos e de Glutationa na Performance e no Metabolismo Muscular Durante a Atividade Prolongada

O fígado, através de seu estoque de glicogênio, é o único órgão capaz de manter constante as concentrações de glicose sanguínea por um certo período de tempo ${ }^{(31)}$. A concentração de glicose circulante é determinada pelo balanço entre a taxa de liberação de glicose pelo fígado e a taxa de consumo desta pelos outros tecidos. Porém, a quantidade de glicogênio estocada no fígado é muito baixa ( 100 g) em relação ao requerimento diário de glicose pelo organismo (>300 $\mathrm{g} / \mathrm{dia})^{(33)}$. Isto requer disponibilidade elevada de glicogênio muscular e de glicose plasmática para a manutenção da performance durante a atividade muscular prolongada ${ }^{(16,19)}$. Há evidências de que o aumento da performance durante o exercício está associado com a manutenção da glicemia e com uma maior taxa de oxidação de glicose no estágio final do exercício prolongado, quando os estoques de glicogênio estão reduzidos ${ }^{(17)}$. Confirmando a hipótese de que a fadiga durante o exercício prolongado coincide com a redução dos estoques intracelulares de glicogênio, vários estudos têm sugerido que o aumento do conteúdo dos intermediários do ciclo de Krebs pela suplementação de carboidratos é fundamental para a maior produção oxidativa de energia durante a atividade física de longa duração $0^{(11,19,20)}$. Uma vez que 0 exercício prolongado resulta em produção elevada de ADP e Pi, seguido de aumento nas concentrações intracelulares de inosina monofosfato (IMP) e amônia ${ }^{(10,11)}$. Isso sugere que a fadiga nesse tipo de atividade está relacionada com uma falha na produção de ATP pela via oxidativa. Por outro lado, a depleção dos estoques de glicogênio muscular e/ou redução da glicemia devido ao comprometimento dos estoques de glicogênio hepático reduz substancialmente a performance durante o exercício prolongado ${ }^{(18,49)}$. A diminuição da concentração de glicogênio resulta em menor disponibilidade de piruvato, um substrato importante para produção de acetil-CoA e para manutenção das vias anapleróticas de produção de intermediários do ciclo de Krebs, incluindo oxalacetato ${ }^{(11,20,33,36,37)}$. Portanto, um aumento do conteúdo dos intermediários do ciclo de Krebs é fundamental para a manutenção da produção de NADH. Em acordo com essa hipótese, Spencer et al. ${ }^{(20)}$, examinando o efeito da suplementação de carboidratos na performance durante a atividade física prolongada, observaram que o conteúdo intramuscular de inosina monofosfato aumenta durante a fadiga em indivíduos com os estoques de glicogênio depletados. Ao passo que, em indivíduos com conteúdo de glicogênio elevado, observou-se um aumento na formação de intermediários do ciclo de Krebs. Apesar dessas observações, alguns estudos não conseguiram demonstrar esse efeito do aumento dos estoques de glicogênio na performance e no conteúdo de intermediários do ciclo de Krebs ${ }^{(50-52)}$.

O glicogênio é o principal substrato da vias das pentoses, como já mencionado, uma via importante de fornecimento de NADPH/glutationa para o sistema glutationa redutase/peroxidase na manutenção do estado redox intracelular ${ }^{(15,23,32)}$, sugerindo que uma menor disponibilidade de glicogênio poderia ainda comprometer a síntese de glutationa, reduzindo a capacidade de defesa antioxidante do músculo esquelético durante contrações prolongadas. Conforme descrito anteriormente, a menor capacidade de manutenção do equilíbrio redox poderia comprometer a atividade de importantes vias metabólicas de produção de energia durante a atividade muscular, incluindo principalmente o ciclo de Krebs e a cadeia de transporte de elétrons mitocondrial(41-45). Portanto, a manutenção da glicemia durante o exercício prolongado é essencial para o melhor desempenho do músculo esquelético(1). Em adição, a glicose éo único substrato energético do sistema nervoso central e das hemácias.
Além das alterações metabólicas induzidas pela redução dos estoques de glicogênio controlar a produção de Espécies reativas de oxigênio durante o exercício prolongado, alterações sistêmicas podem também modificar essa produção. Nesse tipo de atividade, há aumento das concentrações de hormônios relacionados ao estresse como o hormônio adrenocorticotrófico (ACTH), glucagon, cortisol e catecolaminas, seguido da liberação de neutrófilos e monócitos, importantes fontes de produção de Espécies reativas de oxigênio(53). Essas alterações poderiam aumentar a ocorrência de estresse oxidativo durante o exercício prolongado aumentando a liberação de citocinas pró-inflamatórias e ativação de NF-KB, um importante fator de transcrição envolvido na sinalização de diferentes processos celulares ${ }^{(2,6)}$. Porém, o efeito da suplementação de carboidratos na produção de Espécies reativas de oxigênio por essas vias, particularmente durante o exercício prolongado, foi pouco estudado ${ }^{(54)}$. Conforme já descrito, a suplementação de carboidratos contribui com a manutenção da glicemia, reduzindo as concentrações de glucagon, cortisol e catecolaminas no plasma, sugerindo que a suplementação de carboidratos pode reduzir a ocorrência de estresse oxidativo durante o exercício prolongado. Em suporte a nossa proposta nesta revisão, Lord-Fontaine et a ${ }^{(55)}$ mostraram que a suplementação de glicose protege as células do ovário de hamsters contra o estresse oxidativo induzido pelo $\mathrm{H}_{2} \mathrm{O}_{2}$, via aumento do conteúdo de NADPH produzido na via das pentoses.

Em adição ao efeito da suplementação de carboidratos na manutenção da atividade do ciclo de Krebs, na redução da produção de Espécies reativas de oxigênio e na performance, estamos propondo que pode haver um efeito sinérgico desta com a suplementação de glutationa durante a atividade física prolongada. Os resultados dos estudos sobre o efeito da suplementação de glutationa na performance durante o exercício prolongado são controversos. O aumento das concentrações de glutationa na maioria dos tecidos, via suplementação exógena, não é uma tarefa simples, uma vez que glutationa é pouco disponível para a maioria dos tecidos após a administração oral ou por injeção intraperitoneal ${ }^{(1)}$. Para contornar esse problema, o aumento na disponibilidade de cisteína, via suplementação de N-acetil-L-cisteína (NAC), para a biossíntese de glutationa, é uma opção de aumento do conteúdo de glutationa em muitos tecidos ${ }^{(1,3,7)}$. Embora a suplementação de glutationa disponibilize uma quantidade muito baixa desta para os tecidos periféricos ${ }^{(1)}$, têm sido demonstrado que a glutationa aumenta a capacidade de trabalho muscular durante o exercício prolongado em camundongos ${ }^{(56,57)}$. Porém, esses resultados são surpreendentes, uma vez que a suplementação de glutationa tem falhado em aumentar a disponibilidade de glutationa nos tecidos ${ }^{(1)}$, ressaltando a necessidade da realização de novos estudos. Em contraste, animais tratados com a administração aguda de glutationa ou N-acetil-L-cisteína (1 g/kg) e submetidos ao exercício exaustivo, apresentam aumento de estresse oxidativo em vários tecidos, incluindo o músculo esquelético ${ }^{(58)}$. A suplementação de glutationa não protegeu contra o aumento de estresse oxidativo. De modo interessante, a administração de $\mathrm{N}$-acetilL-cisteína protegeu a molécula de glutationa contra oxidação. Porém, a administração de ambos antioxidantes não teve efeito na performance durante o exercício exaustivo de longa duração. É importante salientar que as concentrações de glutationa no músculo esquelético não foram determinadas, indicando que a suplementação pode não ter sido adequada para induzir aumento nas concentrações intracelulares de glutationa no músculo esquelético. A exemplo do armazenamento de glicogênio no fígado, a glutationa é estocada nesse órgão ficando disponível para a manutenção do estado redox dos tecidos ${ }^{(1,7,13)}$. O exercício prolongado reduz os estoques de glutationa, sugerindo que o aumento no conteúdo deste, via suplementação de N-acetil-L-cisteína, pode ser benéfica na proteção contra o estresse oxidativo imposto pela demanda metabólica elevada ${ }^{(1,4,7,13)}$.

O efeito da suplementação de N-acetil-L-cisteína na oxidação de glutationa durante o exercício intenso foi testado em humanos. A su- 
plementação de NAC aumentou a capacidade antioxidante atenuando a taxa de oxidação de glutationa, indicando que o tratamento foi efetivo em manter o estado redox durante a atividade muscular ${ }^{(59)}$. Em adição aos experimentos em que o efeito da suplementação de N-acetil-Lcisteína no estado redox plasmático foi estudado, em experimentos utilizando músculo diafragma de rato foi mostrado que o tratamento com N-acetil-L-cisteína atenua a fadiga muscular durante contrações agudas. Ao passo que, em humanos, a infusão de N-acetil-L-cisteína significativamente aumentou a força de contração muscular durante contrações intensas (fadigante) ${ }^{(60,61)}$.

\section{CONCLUSÕES FINAIS}

Embora estudos conclusivos a respeito do efeito da suplementação de carboidratos e glutationa (NAC) na modulação redox do músculo esquelético durante contrações são escassos, nossa proposta é de que um efeito associado da suplementação de N-acetil-L-cisteína (glutatio- na) e carboidratos deve ser testado. É possível que haja aumento da performance muscular durante a atividade prolongada, aumentando a capacidade de produção de ATP via manutenção da atividade do ciclo de Krebs e equilíbrio do estado redox intracelular. Com base nessa hipótese, estratégias que comprovadamente elevam as concentrações intracelulares de antioxidantes no músculo esquelético, diretamente via suplemetação de $\mathrm{N}$-acetil-L-cisteína (glutationa) ou indiretamente via suplementação de carboidratos, podem aumentar a performance durante o exercício prolongado.

\section{AGRADECIMENTOS}

Este estudo teve suporte financeiro da FAPESP, CAPES e CNPq

Todos os autores declararam não haver qualquer potencial conflito de interesses referente a este artigo.

\section{REFERÊNCIAS BIBLIOGRÁFICAS}

1. Sen CK, Packer L. Thiol homeostasis and supplements in physical exercise. Am J Clin Nutr 2000; 72: 653S-695.

2. Sen CK. Cellular thiols and redox-regulated signal transduction. Curr Top Cell Regul 2000; 36: 1-30.

3. Barreiro E, Galdiz JB, Marinan M, Alvarez FJ, Hussain SN, Gea J. Respiratory loading intensity and diaphragm oxidative stress: N-acetyl-cysteine effects. J Appl Physiol 2006; 100: 555-63.

4. McKenna MJ, Medved I, Goodman CA, Brown MJ, Bjorksten AR, Murphy KT, et al. N-acetylcysteine attenuates the decline in muscle $\mathrm{Na}+\mathrm{K}+$-pump activity and delays fatigue during prolonged exercise in humans. J Physiol 2006; 1:576: 279-88.

5. Ungvari Z, Wolin MS, Csiszar A. Mechanosensitive production of reactive oxygen species in endothelial and smooth muscle cells: role in microvascular remodeling? Antioxid Redox Signal 2006; 8: 1121-9.

6. Sen CK, Packer L. Antioxidant and redox regulation of gene transcription. FASEB 1996; 10: 709-20.

7. Juel C. Muscle fatigue and reactive oxygen species. J Physiol 2006; 1:576:1.

8. Reid MB, Haack KE, Franchek KM, Valberg PA, Kobzik L, West MS. Reactive oxygen in skeletal muscle I. Intracellular oxidant kinetics and fatigue in vitro. J Appl Physiol 1992; 73: 1797-804

9. Reid MB. Redox modulation of skeletal muscle contraction by reactive oxygen and nitric oxide. In: Biochemistry of Exercise X. Ed. M. Hargreaves and M. Thompson, Champaign, IL: Human Kinetics 1999: $185-200$

10. Sahlin K, Tonkonogi M, Soderlund K. Energy supply and muscle fatigue in humans. Acta Physiol Scand 1998; 162: 261-6.

11. Sahlin K, Katz A, Broberg, S. Tricarboxylic acid cycle intermediates in human muscle during prolonged exercise. Am J Physol Cell Physiol 1990; 259: C834-41.

12. Nashiwati E, Dimarco A, Supinski G. Effects produced by infusion of free radical-generating solution into the diaphragm. Am Rev Respir disease 1993; 147:60-5.

13. Gohil K, Viguie C, Stanley WC, Brooks GA, Packer L. Blood glutathione oxidation during human exercise. J Appl Physiol 1988; 64: 115-9.

14. Leeuwenburgh C, Ji LL. Alteration of glutathione and antioxidant status with exercise in unfed and refed rats. J Nutr 1996; 126: 1833-43.

15. Newsholme, EA, Leech AR. Biochemistry for the Medical Sciences. Toronto:Wiley, 1983: 623-627.

16. Hawley JA, Hargreaves M, Zierath JR. Signalling mechanisms in skeletal muscle: role in substrate selection and muscle adaptation. Essays Biochem 2006; 42: 1-12.

17. Spriet, L.L. Regulation of skeletal muscle fat oxidation during exercise in humans. Med Sci Sports Exer 2002; 34: 1477-84.

18. Brooks GA, J Mercier. Balance of carbohydrate and lipid utilization during exercise: the "crossover" concept. J Appl Physiol 1994; 76: 2253-61

19. Claassen A, Lambert EV, Bosch AN, Rodger M, St Clair Gibson A, Noakes TD. Variability in exercise capacity and metabolic response during endurance exercise after a low carbohydrate diet. Int I Sport Nutr Exerc Metab 2005; 15: 97-116.

20. Spencer MK, Yan Z, KatZ A. Effect of low glycogen on carbohydrate and energy metabolism in human muscle during exercise. Am J Physiol. 1992; 262: C975-9.

21. Bangsbo J, Gibala MJ, Howarth KR, Krustrup P. Tricarboxylic acid cycle intermediates accumulate at the onset of intense exercise in man but are not essential for the increase in muscle oxygen uptake. Pflugers Arch 2006; 452: 737-43.

22. Mallet RT. Pyruvate: Metabolic protector of cardiac performance. Soc Exper Biol Med 2000; 223: 136-48.

23. Halliwell B, Gutteridge JM. Iron toxicity and oxygen radicals. In: Free radicals in Byology and Medicine. $2^{\text {nd }}$ Ed. Oxford: Clarendon Press 1989: 86-123.

24. Vina J, Sastre J, Asensi M, Packer L. Assay of blood glutathione oxidation during physical exercise. Methods Enzymol 1995; 251:237-43.

25. Lew H, Pyke S, Quintanilha A. Changes in glutathione status of plasma, liver and muscle following exhaustive exercise in rats. FEBS Lett 1985; 185: 262-6.

26. Jackson MJ, Pye D, Palomero J. The production of reactive oxygen and nitrogen species by skeletal muscle. J Appl Physiol 2007; 102: 1664-70.

27. Silveira LR, Pereira-da-Silva $L$, Juel $C$, Hellsten $Y$. Formation of hydrogen peroxide and nitric oxide in rat skeletal muscle cells during contactions. Free Rad Biol Med 2003; 35: 455-64.

28. Chance B, Sies H, Boveris A. Hydroperoxide metabolism in mammalian organs. Physiol Reviews 1979; 59:527-605.

29. Randle PJ, Garland PB, Hales CN, Newsholme EA. The glucose fatty-acid cycle. Its role in insulin sensitivity and the metabolic disturbances of diabetes mellitus. Lancet I 1963; 13: 785-9.

30. Roberts TJ, Weber JM, Hoppeler H, Weibel ER, Taylor CR. Design of the oxygen and substrate pathways. II. Defining the upper limits of carbohydrate and fat oxidation. J Experim Biol 1996; 199: 1651-8.

31. Jackson MJ, Papa S, Bolanos J, Bruckdorfer R, Carlsen H, Elliott RM, et al. Antioxidants, reactive oxygen and nitrogen species, gene induction and mitochondrial function. Mol Aspects Med 2002; 23: 209-85.

32. Lawler J, Powers S, Criswell D. Inducibility of NADPH-specific isocitrate dehydrogenase with endurance training in skeletal muscle. Acta Physiol Scand 1993; 149: 177-81.

33. Newsholme EA. An introduction to the roles of the glucose-fatty acid cycle in sustained exercise. In: Hargreaves M, Thompson M (Eds).Biochemistry of Exercise IX. Human Kinetics, Champaign, 1999 $119-25$

34. Gibala MJ, Young ME, Taegtmeyer H. Anaplespécies reativas de oxigêniois of the citric acid cycle: role in energy metabolism of heart and skeletal muscle. Acta Physiol Scand 2000; 168: 657-65.

35. Gibala MJ. Anaplespécies reativas de oxigêniois of the muscle tricarboxylic acid cycle pool during contraction: does size matter? J Physiol 2003: 548: 334.

36. Aragon JJ, Lowenstein. The purine-nucleotide cycle. Comparison of the levels of citric acid cycle intermediates with the operation of the purine nucleotide cycle in rat skeletal muscle during exercise and recovery from exercise. Eur J Biochem 1980; 110: 371-7.

37. Owen OE, Kalhan SC, Hanson RW. The key role of anaplespécies reativas de oxigêniois and cataples pécies reativas de oxigêniois for citric acid cycle function. J Biol Chem 2002; 277: 30409-12.

38. Walton $M E$, Ebert $D$, Haller RG. Relative rates of anaplerotic flux in rested and contracted rat skeletal muscle measured by 13C NMR spectroscopy. J Physiol 2003: 548:541-8.

39. Taegtmeyer $\mathrm{H}$, Hems $\mathrm{R}$, Krebs HA. Utilization of energy-providing substrates in the isolated working rat heart. Biochem J 1980; 186: 701-11.

40. Reid MB. Nitric oxide, reactive oxygen species, and skeletal muscle contraction. Med Sci Sports Exerc 2001; 33: 371-6.

41. Andersson $U$, Leighton B, Young ME, Blomstrand E, Newsholme EA. Inactivation of aconitase and oxoglutarate dehydrogenase in skeletal muscle in vitro by superoxide anions and/or nitric oxide. Biochem Biophys Res Commun 1998; 249: 512-6.

42. Tretter $L$, Adam-vizi V. Inhibition of Krebs cycle enzymes by hydrogen peroxide: A key role of [alpha]ketoglutarate dehydrogenase in limiting NADH production under oxidative stress. J Neurosci 2000; 20: 8972-9.

43. Haller RG, Henriksson KG, Jorfeldt L, Hultman E, Wibom R, Sahlin K, et al. Deficiency of skeletal muscle succinate dehydrogenase and aconitase. Pathophysiology of exercise in a novel human muscle oxidative defect. J Clin Invest 1991; 88: 1197-206.

44. Gardner PR, Fridovich I. Quinolinate phosphoribosyl transferase is not the oxygen-sensitive site of nicotinamide adenine dinucleotide biosynthesis. Free Radic Biol Med 1990; 8: 117-9.

45. SuzukiYJ Edmondson JD, Ford GD. Inactivation of rabbit muscle creatine kinase by hydrogen peroxide. Free Radic Res Commun 1992; 16: 131-6.

46. Mohr S, Stamler JS, Brune B. Mechanism of covalent modification of glyceraldehyde-3-phosphate dehydrogenase at its active site thiol by nitric oxide, peroxynitrite and related nitrosating agents. FEBS Lett 1994; 348: 223-7.

47. Cleeter MW, Cooper JM, Schapira AH. Irreversible inhibition of mitochondrial complex I by 1-methyl4-phenylpyridinium: evidence for free radical involvement. J Neurochem 1992; 58: 786-9.

48. Powers SK, Lennon SL. Analysis of cellular responses to free radicals: focus on exercise and skeletal muscle. Proc Nutr Soc 1999: 58: 1025-33.

49. Holloszy JO. Regulation of carbohydrate metabolism during exercise: New Insights and remaining puzzles. In: Hargreaves M, Thompson M. Biochemistry of Exercise IX. Human Kinetics, Champaign 1999: 3-12.

50. Dawson KD, Baker DJ, Greenhaff PL, Gibala MJ. An acute decrease in TCA cycle intermediates does not affect aerobic energy delivery in contracting rat skeletal muscle. J Physiol 2005; 565: 637-43.

51. Howarth KR, LeBlanc PJ, Heigenhauser GJ, Gibala MJ. Effect of endurance training on muscle TCA cycle metabolism during exercise in humans. J Appl Physiol 2004; 97: 579-84.

52. Gibala MJ, Gonzalez-Alonso J, Saltin B. Dissociation between muscle tricarboxylic acid cycle pool size and aerobic energy provision during prolonged exercise in humans. J Physiol 2002; 545: 705-13.

53. McAnulty SR, MCAnulty LS, Nieman DC, Morrow JD, Utter AC, Henson DA, Dumke CL, Vinci DM Influence of carbohydrate ingestion on oxidative stress and plasma antioxidant potential following a 3 h run. Free Radic Res 2003; 37: 835-40.

54. McAnulty SR, McAnulty LS, Nieman DC, Morrow JD, Utter AC, Dumke CL. Effect of resistance exercise and carbohydrate ingestion on oxidative stress. Free Radic Res 2005: 39: 1219-24.

55. Lord-Fontaine $S$, Averill-Bates DA. Heat shock inactivates cellular antioxidant defenses against hydrogen peroxide: protection by glucose. Free Radic Biol Med 2002; 32: 752-65.

56. Novelli GP, Falsini S, Bracciotti G. Exogenous glutathione increases endurance to muscle effort in mice. Pharmacol Res 1991; 23: 149-55.

57. Cazzulani P, Cassin M, Ceserani R. Increased endurance to physical exercise in mice given oral reduced glutathione GLUTATIONA. Med Sci Res 1991; 19: 543-4.

58. Sen CK, Atalay M, Hanninen O. Exercise-induced oxidative stress: glutathione supplementation and deficiency. J Appl Physiol 1994; 77: 2177-87.

59. Sen CK, Rankinen T, Vaisanen S, Rauramaa, R. Oxidative stress after human exercise: effect of $\mathrm{N}$-acetylcysteine supplementation. J Appl Physiol 1994; 76: 2570-7.

60. Khawli FA Reid MB 1994. N-acetylcysteine depress contractile function and inhibits fatigue of diaphragm in vitro. J Appl Physiol 1994; 77: 317-24.

61. Reid MB, Stokic DS, Koch SM Khawli FA, Leis AA. N-acetylcysteine inhibits muscle fatigue in humans J Clin Invest 1994; 94: 2468-74. 